\title{
http://doi.org/10.15359/ree.2004-6.7 \\ MODELOS GERENCIALES EN \\ LA ADMINISTRACIÓN EDUCATIVA
}

Ileana VargasJiménez'

El presente artículo presenta una definición de lo que es la teoría y cuáles son sus funcionés para luego analizar algunos modelos que explican el comportamiento organizacional, y cómo estos afectan en forma positiva o negativa el trabajo de las instituciones educativas. Por último, se señala el modelo denominado Gestión de circulos de calidad y cómo puede influir en la toma de decisiones de las instituciones educativas.
Analysis of various management models that account for organizational behavior and of how these models affect-positively or negatively-the work carried out in educational institutions. The theory and its functions are defined to serve as a basis to the analysis. The paper finishes mentioning the model for the management of quality circles and how it can influence on decision-making processes at educational institutions.

1 Licenciada en Educación Preescolar, con una maestría en Administración Educativa por la Universidad de New México. Estados Unidos. Directora de la División de Educación para el Trabajo del CIDE. 
El artículo tiene como objetivo principal abordar algunos modelos gerenciales que se han desarrollado en las últimas décadas y cómo estos influyen en el campo de la administración educativa. Es importante señalar también el concepto de teoría, y cuál es su función porque existen dudas con respecto a si sirve realmente o no la teoría, el fin principal es comprender la conducta organizacional de las escuelas y cómo los directores la utilizan en forma consciente o inconscicnte.

Primeramente, dcbemos saber que las organizaciones no existen exccpto en la mentc del hombre, son entidades sociales no físicas, tratan de cxplicar cómo cl hombre interacciona efcctivamente para realizar tareas cspecíficas, todo administrador educativo debc utilizar diferentcs destrezas para interpretar y diagnosticar las accioncs de las personas que intcgran las organizaciones educativas, y así proveerse de una gran gama de altemativas para solucionar los problemas que sc le presentan en la institución.

\section{¿Qué es la teoría?}

Scgún Owens (1995, p.15) la teoría cs obscrvada como un proceso mental, una forma de pensar acerca de la realidad para mejor comprender esta última y poderla describir con más precisión. Para aclarar esto, es importantc señalar:

\section{¿Cuáles son las funciones de la teoria?}

Las funciones que se le asignan pueden ser muy útiles y cntre ellas se destacan las siguientes:

- Ayuda a organizar nuestros conocimientos dentro de un conjunto ordenado y sistemático, es decir, en el cáso concreto de la administración educalival, se demuestra en la toma de decisiones, y cómo estas son ejccutadas. Además colabora en el accionar administrativo en relación con los conocimientos que poseen los directores y de acuerdo a su experiencia profesional.

- Proporciona una guía a los investigadores, partiendo del fundamento de que tiene los siguientes elementos: hechos significa que $\mathrm{cl}$ acontecer dentro de una organización se ve envuelta por sucesos que obscrvamos en forma directa; conceptos, quiere decir, que esos hechos se convietten en conceptos cuando se describen ejemplos dc conducta organizacional y sc utilizan tćrminos precisos y descriptivos, y son aceptados por todos los miembros de la institución; hipótesis es aquello que se supone es cierto o que se da por sentado con el propósito de poncr a prueba una suposición, y finalmente, la 
teoría es la relación sistemática que se establece dentro de un grupo general de hipótesis o suposiciones, las hipótesis sobre las cuales se basa una teoría deben haberse verificado hasta el punto de que se acepten generalmente como verdaderas.

- Puede ayudar a explicar fenómenos de conducta organizacional, los cuales serían incomprensibles sin la ayuda de la teoria, quiere decir, que las funciones, son herramientas que ayudan a explicar los fenómenos organizacionales, tales como la comunicación, la motivación de los individuos, las conductas de los participantes, y en fin todo el accionar que se da dentro de las organizaciones.

Para comprender este aspecto de la conducta organizacional, es importante rescatar a diversos especialistas que han venido investigando, especialmente durante las últimas décadas, y que de alguna manera han contribuido a explicar el fenómeno de la teoria.

Los teóricos clásicos formaron ideas nuevas para resolver los problemas administrativos que habian dentro de las organizaciones, su principal exponente fue Frederick Taylor (1856-1915), el enfoque típico de la escuela de la administración científica es el énfasis en las tareas, muchos de ellos fueron definidos como principios científicos y universales porque eran aplicables a toda organización, consideraban que si esos principios se aplicaban, daban como resultado un uso más eficiente del tiempo, de los recursos y de los materiales.

La tcoría clásica suponía que una aplicación de los procesos y estructuras del control administrativo promoveria un comportamiento racional, cficientc y bien disciplinado, por parte de los actores de la organización. El trabajador individual era considerado como un objeto, una pieza de la máquina burocrática.

Sin embargo, los investigadores de las organizaciones se dieron cuenta de que esta teoria no contemplaba al ser humano y por lo tanto fue perdiendo vigencia, posteriormente aparecen otros investigadores tilles como: Chris Argyris, Chester Barnard, Douglas Mc Gregor (Chiavenato, 1992) quienes analizan la teoría organizacional desde diversos aspectos en donde cada uno le agrega conceptos de la siguiente forma:

- Chris Argyris: Se asocia con una teoria organizacional en donde el individuo tiene personalidad y metas las cuales se deben de respetar.

- Chester Barnard: La teoría de la organización debe incluir conceptos tales como la autoridad, toma de decisiones y motivación para que los individuos se sientan satisfechos dentro de la organización. 
- Herbert Simon: La organización tiene que tratar con factores racionales y factores no racionales, es decir, se toman decisiones que en ocasiones no agradan a todos los integrantes de la organización.

Por otro lado, Douglas Mc Gregor, quien ha revolucionado el concepto organizacional, ya que su lcgado intenta explicar ciertos aspectos de la naturaleza del ser humano, describe dos aspectos o modelos a los que denomina teoría X y teoría $\mathrm{Y}$, cada uno de ellos tiene su significado dentro del proceso organizacional. En el siguiente cuadro se ven claramente las diferencias de cada modelo y la manera en que se estudia, así como el impacto que pucde producir en las organizaciones.

\begin{tabular}{c|l}
\hline \multicolumn{1}{c|}{ La teoria X postula } & \multicolumn{1}{c}{ La teoría Y postula } \\
\hline $\begin{array}{l}\text { El ser humano promedio tiene una } \\
\text { aversión innata por cualquier tipo de } \\
\text { trabajo y de ser posible lo evitará. }\end{array}$ & $\begin{array}{l}\text { El gasto de energía que demanda } \\
\text { el esfuerzo físico y mental es tan } \\
\text { natural como el que demanda un } \\
\text { juego o deporte. }\end{array}$ \\
\hline $\begin{array}{l}\text { Por lo que a la mayoría de la gen- } \\
\text { te se le tendrá que obligar, con- } \\
\text { trolar, y hasta amenazar. }\end{array}$ & $\begin{array}{l}\text { La gente practicará la auto-orien- } \\
\text { tación y la autodisciplina hacia las } \\
\text { metas de la organización si se han } \\
\text { comprometido. }\end{array}$ \\
\hline $\begin{array}{l}\text { El ser humano prefiere trabajar } \\
\text { bajo una dirección, desea evitar } \\
\text { responsabilidades, posce poca } \\
\text { ambición y busca seguridad. }\end{array}$ & $\begin{array}{l}\text { El comprometcrsc a lograr los ob- } \\
\text { jetivos es una función de recom- } \\
\text { pensa asociada con sus logros. }\end{array}$ \\
\hline
\end{tabular}

\section{Teoría de Douglas Mac Gregor}

Robert Ovens, Comportamiento organizativo en la escuela.

Es asi como la teoría $\mathrm{X}$ presenta un panorama más pesimista y rigido, donde el control es realizado de mancra impucsta por el director, mientras que la teoría y representa todo lo contrario, es más optimista, tiende a la motivación del individuo y procura un ambiente flexible y dinámico, procurando el logro de la autodirección y la integración de los requerimientos individualcs con las necesidades y exigencias plantcadas en la organización. 
Chris Argyris retoma el modelo de Douglas Mc Gregor y le hace un ajuste en el sentido de que habla de patrones de conducta pero sin dejar de lado la teoría X y la teoría $Y$, el cual se señala en el cuadro

\begin{tabular}{l|l}
\hline $\begin{array}{l}\text { Las características de la teoría X } \\
\text { dan lugar al surgimiento del } \\
\text { patrón de conducta A. }\end{array}$ & $\begin{array}{l}\text { El patrón de conducta B } \\
\text { considerado flexible. }\end{array}$ \\
\hline $\begin{array}{c}\text { Y puede tomar algunas de las } \\
\text { siguientes características: } \\
\text { considerado inflexible, se } \\
\text { caracteriza por un liderazgo } \\
\text { enérgico que no acepta ningún } \\
\text { comportamiento irregular y } \\
\text { ejerce controles severos y de } \\
\text { supervisión. }\end{array}$ & $\begin{array}{l}\text { Incluye una gran dosis de } \\
\text { persuasión que se gana la } \\
\text { sumisión o docilidad de los } \\
\text { subordinados, se establecen } \\
\text { buenas relaciones humanas. } \\
\text { Se tiene clara la intención de } \\
\text { manipular, controlar y dirigir. }\end{array}$ \\
\hline
\end{tabular}

Ante tales planteamientos surge una pregunta que es necesario rescatar: ¿Cómo influye esas teorías y postulados a la administración escolar? Para aclararlo se muestra el siguiente cuadro, en donde se presentan dos aspectos, uno es en relación las personas y el otro es en relación con la participación en las instituciones educativas.

\section{En relacion con las personas}

- Los docentes comparten un conjunto de necesidades al pertenecer a un grupo, ser aceptado, ganarse el respeto.

- Lo más importante para ellos es sentirse útiles dentro de la escuela, donde ofrecen sus servicios.

- Tienden a cooperar y a observar las metas que se han fijado tanto la escuela como las del resto.
En relación con la participación

- La tarea básica de un adminis. trador es convencer a cada maestro de que es un elemento importante y útil al equipo de que forma parte.

- El administrador está deseoso de explicar sus decisiones y de discutir las objeciones que le puedan plantear, se les alienta a planificar y a tomar decisiones. 
- Descan contribuir en forma cfectiva y creativa al logro de los objetivos que valgan la pena.

- Lal mayoría de docentes son capaces de desarrollar mucha más creatividad y responsabilidad que lo que Ic permite su empleo.

- Estas aptitudes representan recursos aún sin aprovechar $y$ que se están desperdiciando en el presente.
- La tarea básica del administrador cs crear un medio ambiente en el cual los macstros puedan contribuir con toda su capacidad.

- El administrador permitc y alienta a los maestros a participary a tomar decisiones no sólo en asuntos rutinarios sino en asuntos de mayor importancia.

Ileana Vargas, CIDE UNA, Heredia.

Dentro de las organizaciones educativas el director debe conocer estas teolías y tratar de ajustarlas a su tarea diaria, especialmente para hacer de su tarea un trabajo más compctitivo, real y acorde a las necesidades de su institución.

Actualmente, se desarrollan algunos modelos gerencialcs que toman en cuenta los aspectos que se han scñalado a lo largo del artículo, sin cmbirgo, sc retomará parà cfectos del presentc análisis el modclo denominado Gestión de la calidad total.

\section{Origenes de la Gestión de la calidad total}

El concepto de callidad total surgió en el mundo empresaltial, concretamente en Japón, como control de callidad total, luego pasó a los Estados Unidos y' más tarde a Europa, no obstante los primcros estudios sobre el control de calidad sc llevaron a cabo en Estades Únidos. A partir de los años cincuenta y hasta la décalda de los seicntiı el concepto de calidad ticne un gran desarrollo en Japón scgín Ishikan:a (199.5) con E.W. Deming y J.M. Juran quicnes contribuycron en el sistema integráa cle gestión de la callidad. Éste se trata cle un sistema y cstratcgial de gestión que implica a todo el personal de la empresa en la mejora continua de la calidad de los productos y de los servicios.

Es importinte conocer los fundamentos epistemológicos, esto porque nos servirái palıa poder compicnder cómo sc gestó cstc modelo y' sus posibles implicaciones en cl ámbito cducalivo. 


\section{Fundamentos epistemológicos e ideológicos de la Gestión de la calidad total}

Se mezcla de diversos retazos de leorías, responde más bien a los postulados de la teoría de los recursos humanos, pucs mientras los teoricos de las relaciones humanas consideraron que personalidad y organización estaban en un conflicto sin solución y defendieron el lado humano de la organización, los teoricos de los recursos humanos considerarán que dicho conflicto no es inherente a la organización, ni inevitable. Se defiende así la posibilidad de integrar ambos componentes de tal manera que el hombre logre un máximo de satisfacción y enriquecimiento a partir de su trabajo y al tiempo se obtengan nuevos niveles de eficacia en la organización.

Por lo que los círculos de calidad sc definen como un conjunto articulado de elementos de la escuela de relaciones humanas, de la perspectiva sistémica de las organizaciones, y de la cultura organizativa, la gestión de calidad total (G.C.T) como se le conoce también, produce un sistema de valores que conjuga los valores personales de sus funcionarios con los valores de los productos de la empresa, en el que la calidad ocupa un lugar destacado. Este sistema de valores se convierte en la cultura organizativa de la institución, procura integrar a los trabajadores en la empresa, colocándola como mediadora entre aquellos y la sociedad.

Para poder comprender mejor qué son los círculos de calidad, se agregará la definición de la UNESCO (200l), ya que sc ha dado a la tarca de investigar la calidad y su posible utilización en cl campo de la cducación.

- "La calidad se relaciona con los factores que influyen en clla, en cl campo de la cducación con el currículum, los docentes, los materialcs educativos, la infracstructura y otros. Además la medición de los resultados educativos entre los que sc destacan la descrción, y la repetición cscolar. Sin dejar de lado, cl aspecto del liderazgo, las cstraltcgias, y lis políticas, la gestión de personal, los recursos, y los procesos, la salisfacción de los clientes, la satisfacción del personal, cl impacto en la socicdad y los resultados, componentes que constituyen las grandes metas de la organización, pero se propone una definición adaptada a las instituciones cducalivals: el producto cducativo, la salisfacción de los alumnos, la satisfacción del personal del centro, cl discño de la cstratcgia, la gestión de recursos humanos y matcriales, la metodología cducativa" (pág 13).

Como sc pucde apreciar cs un concepto que abarca a todo el quchacer dcl sistemal clucalivo, no solamentc a un solo clemento, se ve a todo cl conglomerado institucional. 


\section{Gestión de calidad total en educación un nuevo modelo organizativo}

Desde hace pocos años se presenta la gestión de calidad total como una estrategia para la mejora de la calidad y en algunos casos incluso como la estrategia por excelencia, y como liı G.C.T. no surgió en el campo educativo se hace nccesario preguntarse: ¿cuáles son los fundamentos teóricos y los procedimicntos básicos de esta nueval forma de enfocar la gestión organizativa?, y proceder a su análisis. Es necesario preguntarse: ¿cómo se adecua la gestión de la calidad total al campo educativo?

Scgún revistas educativas data desde 1995 en diversos trabajos sin embargo, se introduce algo más temprano en Brazil y Portugal, e igual en Nortcaméricil en 1990.

\section{Implicaciones del uso de la calidad total en educación}

Es necesario señalar que las implicaciones de este modelo en educación debc conllevar a lo siguiente:

- La implantición de un sistema de G.C.T. requiere de cvaluadores cxternos, quicnes monitorcan cl proceso en los centros cducativos.

- Algunos investigiadores reconocen que para aplicar la G.C.T. en las escuelas éstas deben rcunir cicrtas condiciones, se fundamenta en una visión y un trabajo en equipo absolutamente compartido.

- La autonomia es considerada un elcmento clave parai cl desarrollo de la C.T.E.

\section{Conclusión}

El conoccr los modelos que se han venido desiurtollando en los últimos años nos permiten comprender y articular mejor el desarrollo de las organizalciones, es decir; no pueden funcionad aisladals ni separadals del mundo extemo, los modelos nos indicin que paral trabaijar más adecualdamentcy aprovechar los recursos instilucionalcs, humalnos y financicros es necesanio comprender y mancjar esas tcorias.

Es importante mencionar que no haly ningún modelo mejoro peor que otros. simplemente explicàn el fenómeno organizacional, lo importante es conocerlos y tratalt de que nucstro trabiljo scil más llexible y brinde resultados saltisfactorios a los que integran la institución. Con respecto al los circulos de cillidald, significal crear conciencia de callidald en todos y calda uno de los micmbros de una organización a través del trabajo en cquipo y el intercambio de cxperiencias y conocimicntos, proponiendo ideas y alternaltivis con un enfoque de mejora continual. 


\section{Referencias}

Chiavaneto, I. (1990). Administración de Recursos Humanos.

Chiavaneto, I. (1992). Introducción a la Teoría de la Administración, ( $3^{2}$ ed.), México: Editorial Mc Graw Hill.

Ishikawa, K. (1995). Qué es Control total de Calidad? Bogotá: Grupo Editorial Norma.

Owens, R.(1995). Comportamiento Organizativo en la Educación.

Sergiovanni T. (1989). Perspectivas de la Supervisión Educativa. Estados Unidos: Mc Graw Hill.

UNESCO. Conferencia Mundial sobre Educación Supcrior (2001).

Vargas I. (2000). Documentos elaborados para el curso de Teorías de la Administración Educativa. ClDE-UNA. 